\title{
Criminologie
}

\section{L’enjeu des années 1990 : la police, les tribunaux et les municipalités}

Irvin Waller

Volume 23, numéro 2, 1990

Après le crime : survivre

URI : https://id.erudit.org/iderudit/017296ar

DOI : https://doi.org/10.7202/017296ar

Aller au sommaire du numéro

\section{Éditeur(s)}

Les Presses de l'Université de Montréal

ISSN

0316-0041 (imprimé)

1492-1367 (numérique)

Découvrir la revue

Citer cet article

Waller, I. (1990). L'enjeu des années 1990 : la police, les tribunaux et les municipalités. Criminologie, 23(2), 89-105. https://doi.org/10.7202/017296ar
Résumé de l'article

This article looks at the 1990's agenda for Québec to improve the protection for victims of crime.

It summarises the progress made by Québec in implementing the United Nations Declaration on Victim Rights. It compares the progress in Québec with other provinces and countries.

It examines how police leadership to implement procedures that respect victims of crime could help the police, while improving significantly respect for victims. It discusses how Québec could combine the civil and criminal interests of victims of crime, while reducing court backlogs. It stresses the importance of reducing victimisation by building on the Agenda for Safer Cities developed in Montréal in 1989. It also calls for more comparative research on the extent to which reforms in different jurisdictions are meeting the needs of crime victims. 


\section{L'ENJEU DES ANNÉES 1990: \\ LA POLICE, LES TRIBUNAUX ET LES MUNICIPALITÉS \\ Irvin Waller*}

This article looks at the 1990's agenda for Québec to improve the protection for victims of crime.

It summarises the progress made by Québec in implementing the United Nations Declaration on Victim Rights. It compares the progress in Québec with other provinces and countries.

It examines how police leadership to implement procedures that respect victims of crime could help the police, while improving significantly respect for victims. It discusses how Québec could combine the civil and criminal interests of victims of crime, while reducing court backlogs. It stresses the importance of reducing victimisation by building on the Agenda for Safer Cities developed in Montréal in 1989. It also calls for more comparative research on the extent to which reforms in different jurisdictions are meeting the needs of crime victims.

Comment le Québec peut-il améliorer l'aide aux victimes d'actes criminels? Comment les cours criminelles peuvent-elles aussi rendre justice aux victimes? Comment faire en sorte qu'il y ait moins de victimes? À qui revient l'initiative dans ce domaine? Quel est le rôle des décideurs au sein des services de police, de l'appareil judiciaire et des municipalités?

Depuis 25 ans, le taux de victimisation criminelle a plus que doublé au Québec. Chaque année, un ménage sur 25 sera victime d'une introduction par effraction, ce qui correspond en moyenne à trois cambriolages par foyer au cours d'une durée de vie normale; une femme sur trois sera victime d'agression sexuelle au cours de sa vie; les femmes, les enfants et les personnes âgées seront agressés dans leur propre maison (USA 1988; CCDS, 1984; Canada, 1983b).

Heureusement, on remarque aussi depuis ces 25 dernières années une sorte de «révolution tranquille» visant à répondre aux besoins des victimes d'actes criminels (Waller, 1983; Canada, 1983; CCDS, 1985 et 1981). L'État indernnise les victimes qui en font la demande, on renseigne les victimes sur

* Professeur, Département de criminologie, Université d'Ottawa, Pavillon Tabaret, Ottawa, Ontario, KIN 6N5. 
le processus de justice pénale, leur participation au processus judiciaire ne se limite pas toujours au rôle de témoin, elles peuvent être dédommagées à l'occasion et bénéficier des services de bénévoles qui les aident à surmonter leur traumatisme. Les maisons d'hébergement et les centres d'aide pour les victimes d'agression sexuelle se multiplient dans les grandes villes. On a instauré de nouveaux programmes et adopté des mesures législatives pour les enfants, telle la Loi sur la protection de la jeunesse (Québec, 1979).

Mais il reste encore beaucoup à faire. La police demeure le service le plus sollicité par les victimes, mais elle manque de lignes directrices sur la façon de traiter ces dernières, de les informer des ressources existantes ou de les diriger vers des services d'aide. Même si le processus pénal a un effet direct sur la réparation des préjudices, de même que sur la sécurité et la vie privée des victimes, il ne prévoit guère de mécanismes permettant à ces dernières d'exprimer leur point de vue. On pourrait aussi faire beaucoup plus en matière de prévention.

\section{LA CHARTE DES NATIONS UNIES POUR LES DROITS DES VICTIMES D'ACTES CRIMINELS}

En 1985, l'Assemblée générale des Nations Unies a adopté une charte pour défendre les droits des victimes d'actes criminels, la Déclaration des principes fondamentaux de justice relatifs aux victimes de la criminalité et aux victimes d'abus de pouvoir (Bassiouni, 1988; ONU, 1985). Elle indiquait ainsi au monde entier quelies mesures les États et les sociétés pouvaient prendre pour remédier aux préjudices, aux pertes et aux traumatismes subis par les victimes d'actes criminels. La déclaration de l'ONU rappelle que les souffrances vécues par les victimes restent souvent négligées ou oubliées et sont aggravées par un système judiciaire d'abord conçu pour punir les contrevenants. On n'aide guère les victimes à obtenir un dédommagement et à surmonter le stress post-traumatique (Waller, 1985). Les familles et les amis des victimes en souffrent également, sans oublier les personnes qui les assistent, comme les policiers. La déclaration des Nations Unies établit quatre grands principes à suivre pour corriger la situation (paragraphes 1-17), recommande des proscriptions additionnelles en matière d'abus de pouvoir et offre des solutions pour les victimes d'autres violations des normes internationales des droits de la personne (paragraphes 18-21).

Le premier principe préconise des moyens permettant aux victimes d'avoir accès aux procédures judiciaires et administratives, de recevoir de l'information et de bénéficier d'un traitement équitable; il insiste aussi sur l'importance de tenir compte de leur opinion lorsque leur intérêt personnel 
est en jeu (paragraphes 4-7). Le second principe propose une série de mesures, comme la médiation, pour favoriser le dédommagement des victimes par les contrevenants (paragraphes 8-11). Le troisième principe demande aux États d'indemniser les victimes lorsque les contrevenants ne peuvent les dédommager de manière satisfaisante (paragraphes 12-13). Enfin, le quatrième principe insiste sur l'aide à apporter à la victime en spécifiant quel type de soutien elle doit recevoir de la part des intervenants (paragraphes 14-17). Un paragraphe entier (paragraphe 16) insiste sur le besoin de former le personnel «des services de police, de l'administration de la justice, des services de santé, des services sociaux et autres... pour les sensibiliser aux besoins des victimes» et préconise l'adoption de «lignes directrices pour assurer une aide adéquate et rapide».

Dans sa résolution, l'Assemblée générale des Nations Unies recommande diverses stratégies d'application de ces principes ainsi que des mesures pour rêduire la victimisation (préambule, 4-9), mesures qui vont de l'adoption de politiques sociales et économiques et de programmes en matière de santé et d'éducation, jusqu'à la collaboration entre les États dans la poursuite des contrevenants.

Toutes ces mesures concernent la victimisation dans les lieux publics ou à l'intérieur du foyer, et s'accompagnent de dispositions spéciales pour les personnes les plus vulnérables comme les femmes, les enfants, les personnes âgées et les membres des minorités raciales. La définition de la victime d'acte criminel donnée par les Nations Unies met l'accent sur les souffrances résultant d'une victimisation et prévoit l'application des mêmes mesures aux victimes d'abus de pouvoir.

Un premier geste pour inscrire les principes des Nations Unies dans la législation canadienne, a été posé en 1988 alors que le gouvernement fédéral, les provinces et les territoires se sont mis d'accord sur des principes de justice pour les victimes d'actes criminels visant à favoriser leur accès au système judiciaire, à leur assurer un traitement équitable et à leur offrir des services d'aide.

La loi C-89 (1989) a été adoptée pour favoriser le recours au dédommagement, l'utilisation de la déclaration de la victime et l'imposition d'amendes spéciales aux contrevenants destinées à financer les services d'aide aux victimes. Toutefois, les principes de l'ONU n'y ont pas été inclus. En revanche, la plupart des provinces les ont inscrits dans leurs propres législations. 


\section{LA SITUATION DU QUÉBEC PAR RAPPORT AUX AUTRES PROVINCES}

Le Québec (1989) a pris de nombreuses mesures pour appliquer les principes des Nations Unies. Certains de ces changements ont mis l'accent sur des types particuliers de victimes (par exemple, les victimes de violence conjugale). D'autres mesures s'adressent plus particulièrement aux enfants et enfin, certains changements concernent toutes les victimes d'actes criminels.

Voyons quelles ont été les principales initiatives québécoises des dernières années dans ce domaine et de quelle façon elles se comparent aux programmes des autres provinces.

En 1972, le Québec a instauré à l'échelle de la province un programme d'indemnisation des victimes de crimes contre la personne. Même si la plupart des provinces possèdent de tels programmes, le Québec indemnise plus les victimes que toutes les autres provinces réunies (Canada, 1986), car il utilise les critères appliqués pour les accidentés du travail.

En 1977, le Québec a adopté une loi générale sur la protection de la jeunesse combinant le bien-être de l'enfant à la protection de ses droits. En 1983, la loi fédérale sur les jeunes contrevenants a établi des principes similaires.

En 1979, les ministères de la Justice, de la Santé et des Services sociaux ainsi que le Conseil du statut de la femme ont organisé dans la province des colloques sur la violence familiale; ces ateliers ont mené à la création de nombreuses tables de concertation qui ont rapproché les divers organismes susceptibles d'aider les victimes de violence familiale. On préconise généralement ce genre d'organismes de coordination ailleurs au Canada, mais un grand nombre de collectivités n'en ont toujours pas (MacLeod, 1989).

En 1974, la ville de London, en Ontario, mettait sur pied son célèbre programme d'intervention policière qui fait appel à des travailleurs en santé mentale dans les cas d'enfants maltraités, de violence conjugale ou d'autres situations de crise. Ces unités interviennent dans les querelles familiales et s'occupent des soins et de la prise en charge des enfants. Elles s'occupent également des personnes dépressives et suicidaires ainsi que des personnes âgées. Leur originalité réside dans leur capacité à intervenir auprès des personnes en crise et à les diriger vers les organismes pouvant leur offrir de l'aide à plus long terme. En 1979, cette initiative a mené à la création d'un comité de coordination interagences qui a favorisé la mise sur pied d'un centre pour les femmes violentées et de nombreux autres services et programmes axés sur la violence familiale. 
En 1982, le Québec mettait sur pied INFOVAC, un système d'information à l'échelle de la province qui renseigne les victimes sur l'issue de leur cause: mise en accusation, tenue des audiences, verdicts et sentences.

Certains services policiers, comme ceux de Calgary et d'Edmonton (1989; Liaison, 1982) avaient déjà institué en 1979 des protocoles à l'intention des agents patrouilleurs permettant d'informer les victimes. Depuis, ils se sont dotés d'unités spécialisées pour intervenir auprès des victimes. Au début des années 1980, le Manitoba a créé dans ses tribunaux un programme à l'intention des victimes et des témoins.

En 1983, 400 personnes ont participé à un important colloque sur les victimes d'actes criminels et la collectivité, organisé par la Société de criminologie du Québec (Rizkalla, 1984). D'autres provinces, comme l'Ontario, la Colombie-Britannique et le Manitoba, ont organisé des rencontres similaires. En 1989, l'Alberta a tenu une rencontre nationale destinée à la mise sur pied d'une «Association canadienne d'assistance aux victimes».

En 1984, le Québec a publié un ensemble de procédures d'intervention auprès des victimes d'agression sexuelle. Cette initiative résultait de la collaboration des ministères des Affaires sociales et de la Justice avec le Conseil du statut de la femme et le milieu médical. On y trouvait une trousse médicolégale conçue pour répondre à la fois aux besoins médicaux de la victime et aux exigences des tribunaux. D'autres provinces ont adopté des programmes similaires.

En 1984, un regroupement d'organismes québécois d'aide aux victimes a créé une association provinciale appelée «Plaidoyer-Victimes» qui s'est avérée une source inépuisable d'énergie, d'encouragement et d'idées quant à l'amélioration des programmes gouvernementaux et de l'intervention des groupes auprès des victimes d'actes criminels. Il n'existe aucune association similaire dans les autres provinces, bien qu'on y trouve des associations de victimes telles que le regroupement des parents d'enfants assassinés (Amernic, 1984), les Canadiens préoccupés par les actes criminels à l'égard des enfants et les victimes de conducteurs ivres. Ces associations remplissent un rôle important tant sur le plan de la définition de priorités politiques à l'égard des victimes d'actes criminels que sur le plan du soutien apporté aux victimes.

En 1984, Micheline Baril, chercheure, intervenante auprès des victimes, militante et organisatrice chevronnée a reçu le prestigieux Prix Stephen Schafer décerné par la United States National Organization for Victim Assistance (NOVA). Elle demeure la seule personne non américaine à avoir reçu ce prix qui récompense l'excellence en matière de recherche et d'action combinées. Le département de police d'Edmonton s'est vu gratifier d'un prix similaire 
de la part de NOVA pour son assistance pratique aux victimes d'actes criminels.

En 1986, le Québec, à l'instar d'autres provinces, a pris des mesures pour que la police poursuive un plus grand nombre de conjoints violents. En 1987, le ministère de la Justice et celui du Solliciteur général du Québec ont organisé des consultations dans toute la province sur l'assistance aux victimes. L'année suivante, le Québec a adopté sa Loi sur l'aide aux victimes d'actes criminels. Cette loi inscrivait les droits et les responsabilités des victimes d'actes criminels dans la législation provinciale, créait un fonds spécial ainsi qu'un bureau d'assistance aux victimes au sein du ministère de la Justice. Ce bureau est responsable de la mise sur pied des centres locaux d'aide aux victimes et supervise l'application des principes inscrits dans la loi.

Le Manitoba (1986) et le Nouveau-Brunswick ont adopté des lois similaires en 1986. La plupart des provinces et des territoires possèdent maintenant des lois de ce genre qui vont d'ailleurs plus loin que celle du Québec parce qu'elles prévoient une suramende compensatoire pour les infractions de juridiction provinciale ainsi qu'un comité indépendant qui recommande de quelle façon utiliser ces fonds. On demande généralement aux organismes représentant les victimes d'actes criminels, aux services de police, aux procureurs de la défense et de la couronne d'être membres de ce comité.

De nombreuses municipalités ontariennes offrent de l'aide aux victimes par l'intermédiaire des services de police et des programmes pour les victimes qui témoignent en cour. L'Ontario n'a pas encore adopté de loi sur les victimes d'actes criminels, mais le gouvernement a proposé une loi sur les services de police qui fait de l'aide aux victimes une des priorités de la police (Ontario, 1989).

En 1987, le Québec a adopté des politiques pour renforcer l'efficacité des poursuites contre les personnes accusées d'abus sexuels sur des enfants. On veut par exemple expliquer aux enfants appelés à témoigner à la cour leur rôle lors du prucès. On a également voulu encourager l'utilisation des enregistrements magnétoscopiques lorsque cela s'avère nécessaire. Ceci permet d'éviter aux enfants de répéter plusieurs fois leur témoignage.

D'autres provinces ont pris des initiatives similaires. L'Ontario a mis sur pied d'importants projets de recherche pour améliorer le traitement des enfants victimes d'abus sexuels qui doivent comparaître comme témoins.

À la fin des années 1980, on a pu voir se développer au Palais de Justice de Montréal de nombreuses initiatives en matière de recherche et de projets. Par exemple, on a pris des mesures spéciales pour que la couronne et la défense s'entendent sur la communication de la preuve avant procès, afin d'économiser 
du temps et d'éviter des stress inutiles pour tout le monde. C'est également dans ce Palais de Justice que l'on a instauré avec le plus de succès au Canada l'utilisation systématique des déclarations des victimes. (Baril et Cusson, 1989).

\section{L'AIDE AUX VICTIMES DANS DIVERS AUTRES PAYS}

Depuis dix ans, des pays comme les États-Unis, la Grande-Bretagne, l'Australie du Sud et la France ont procédé à des réformes radicales afin de mieux répondre aux besoins des victimes. Certaines de ces mesures concernent le droit des victimes à participer au processus pénal; d'autres visent à améliorer l'aide financière et sociale apportée aux victimes.

Les États-Unis ont adopté plus de 1500 dispositions législatives pour protéger les victimes d'actes criminels (NOVA, 1989). Elles comprennent souvent une charte des droits des victimes qui définit de quelle manière les services policiers, les tribunaux et les organismes communautaires doivent traiter les victimes. Certaines mesures cherchent à consolider le financement des services aux victimes par l'imposition de suramendes compensatoires. D'autres visent l'amélioration des services destinés à des groupes spécifiques, comme les femmes battues ou les enfants victimes d'abus sexuels.

L'État du Massachussetts, avec sa population d'environ six millions d'habitants, est un bon exemple de ce genre d'initiatives. Il collecte chaque année plus de quatre millions de dollars pour les coûts des services d'aide où les intervenants écoutent les victimes, leur donnent de l'information et les aident à défendre leurs droits.

Les services auprès des victimes sont surtout développés dans les bureaux des procureurs de la Couronne, et dans les organismes communautaires s'adressant aux femmes et aux enfants violentés. Ces services sont généralement dispensés par des professionnels.

Dans plusieurs États, des dispositions législatives accordent à la victime le droit de faire connaître au tribunal, en audience ou par écrit, le préjudice qu'elle a subi et ses besoins en matière de réparation.

Dernièrement, les associations locales de défense des droits des victimes, comme les groupes de parents d'enfants assassinés, ont réussi à s'organiser en coalitions pour faire inscrire dans les constitutions des États des amendements spécifiant les droits des victimes. Ce mouvement en plein essor exige des responsables des corps policiers, de l'administration de la justice et des services sociaux, la mise sur pied des services mentionnés dans les chartes des droits. 
L'Angleterre et le pays de Galles ont pris d'importantes mesures afin que chaque collectivité soit dotée d'un service d'aide aux victimes. Ces services sont administrés par un coordonnateur salarié qui forme et supervise des bénévoles pour aider les victimes d'actes criminels, en particulier les victimes de vols par effraction. Suite à une évaluation de ce travail (Maguire et Corbett, 1987), le gouvernement a octroyé un budget supplémentaire de plus de 18 millions de dollars pour consolider et étendre ces services à une clientèle plus large de victimes, à partir du moment où le crime a été commis jusqu'au procès final et même au-delà. La répartition de ces fonds est confiée à un comité associé à la National Association of Victim Support Schemes.

En 1964, l'Angleterre a suivi l'exemple de la Nouvelle-Zélande en amorçant un programme d'indemnisation des victimes de violence criminelle. Bien que les victimes ne soient pas aussi bien informées de l'existence de ce programme que dans d'autres pays, aucun plafond aux montants des indemnisations n'a été fixé. Les quelques victimes gravement handicapées reçoivent ainsi des montants presque équivalents à ceux qu'elles auraient obtenu devant les tribunaux civils. Dans une étude célèbre sur le suivi des victimes d'actes de violence pendant les trois années après l'infraction, Shapland (1985) suggère plusieurs moyens visant à mieux adapter les services de la commission d'indemnisation aux besoins des victimes. Ce programme d'indemnisation verse environ $25 \%$ de plus per capita que ce que versera le Québec cette année.

La France a multiplié ses efforts afin que les victimes soient bien informées de leurs droits. Elle a élaboré des procédures précises à l'intention des policiers et du ministère public. La France compte plus de 80 organismes d'assistance aux victimes - regroupés dans l'Institut national d'aide aux victimes et médiation (INAVEM, 1986) - qui coopèrent avec la police et les procureurs pour aider les victimes à se rétablir du traumatisme psychologique et à obtenir l'aide financière ou autre à laquelle elles sont admissibles (De Liège in Études Pénales, 1988). La France a fait de la prévention du crime une priorité nationale (Waller, 1989a). Plus de 500 commissions municipales de prévention du crime ont été implantées avec succès. Outre la prévention du crime, ces commissions cherchent également à améliorer les services offerts aux victimes d'actes criminels.

L'Australie du Sud est le premier État qui a mis en application la Déclaration des Nations Unis dans sa juridiction. Elle a endossé 17 principes en 1986 (Bassiouni, 1988). D'autres États australiens ainsi que la NouvelleZélande adoptent présentement des dispositions similaires en conformité avec la Déclaration de l'ONU. L'Australie du Sud a été également le premier pays 
de Common Law à établir un programme de prévention du crime selon le modèle de la France.

\section{COMMENT RÉPONDRE AUX BESOINS DES VICTIMES DANS LES ANNÉES 1990}

Il y a trois secteurs dans lesquels le Québec a nettement besoin d'améliorations. Les policiers doivent faire plus pour les victimes - comme on le fait dans d'autres régions canadiennes; il faut attribuer aux victimes un rôle plus grand dans la procédure pénale - comme le font les États-Unis et la France, et si on se réfère au programme «Pour des villes sûres» (FCM, 1990) et au modèle français, il faut instaurer des programmes pour prévenir la victimisation.

\section{FAIRE DE LA POLICE UN SERVICE D'AIDE DE PREMIÈRE LIGNE}

Lorsque les victimes appellent la police, il s'agit très souvent de leur première prise de contact avec le système de justice pénale. De plus, les victimes ont souvent besoin de soins médicaux, de protection et de support pouvant être offerts par des services d'aide d'urgence. Enfin, lorsqu'elles se font voler des biens, les victimes désirent les récupérer.

Les policiers sont disponibles 24 heures par jour, sept jours par semaine et disposent de moyens de communication sophistiqués reliês à un répartiteur central. Cet équipement peut leur permettre de se renseigner et de localiser très rapidement les services disponibles; on pense par exemple aux urgences, aux centres d'aide aux victimes d'agression sexuelle, aux maisons d'hébergement pour femmes victimes de violence conjugale, aux centres d'aide pour les victimes et aux commissions d'indemnisation des victimes.

Les victimes veulent obtenir des renseignements sur le contrevenant, (connaître la date de sa libération par exemple) et suivre les progrès de l'enquête policière. Lorsque l'infracteur a été identifié, il se peut que les victimes cherchent à savoir si celui-ci risque d'exercer des représailles. Elles veulent aussi savoir ce qui s'est passé lors des enquêtes sur cautionnement et désirent parfois donner leur opinion. Elles se montrent souvent extrêmement surprises en apprenant qu'un contrevenant qui vient d'être arrêté a été aussitôt relâché. Souvent, les victimes veulent aussi être informées sur les mesures à prendre pour se prémunir contre de nouveaux préjudices, comme par exemple sur la façon d'assurer la protection de leur maison. Il est donc important d'offrir aux victimes un service d'information fiable sur les mesures de protection qu'elles peuvent prendre. 
Pour les policiers, la plupart des crimes impliquent une victime. C'est la victime qui, le plus souvent, signale une infraction criminelle et qui donne une description du crime et du suspect. La coopération de la victime facilite l'arrestation et la condamnation des infracteurs. En outre, la satisfaction des victimes peut avoir un effet favorable sur l'opinion publique au moment où on vote les budgets alloués aux services de police (Waller, 1990; Waller, 1989b).

La police d'Edmonton, en Alberta, possède le programme d'aide aux victimes le mieux rodé, avec près de 3000 victimes bénéficiant chaque mois d'une forme ou une autre de communication (Edmonton, 1989; Liaison, 1982; Pullyblank, 1986).

Certaines villes ont mis sur pied des programmes spéciaux d'intervention dans les cas de violence familiale, comme London, en Ontario, où on peut faire appel 24 heures sur 24 à des équipes de policiers et de travailleurs sociaux (Jaffe, 1984).

L'Ontario a dernièrement amendé sa Loi sur les Services de police (Ontario 1989) afin de préciser les responsabilités des cadres et des agents de la police et de répondre ainsi «à la nécessité d'assurer la sécurité de toutes les personnes et tous les biens en Ontario» et «à la nécessité de respecter les victimes d'actes criminels et de comprendre leurs besoins». Étant donné l'importance de la collaboration de la victime, on pourrait s'attendre des responsables des corps policiers qu'ils fassent en sorte que les victimes soient traitées comme des «clients privilégiés» et qu'ils appliquent les politiques de l'Association internationale des chefs de police qui demandent aux services de police «d'élaborer des protocoles et de former le personnel» afin de respecter les «droits inaliénables de toutes les victimes de crime», à savoir:

«le droit à la protection contre toute intimidation;

le droit d'être informé sur l'aide financière et les services sociaux accessibles et des moyens de s'en prévaloir;

le droit d'être protégé lors des entrevues et des audiences, et dans les cas où la présence de la victime en cour n'est pas nécessaire, le droit d'en être averti;

le droit à une restitution rapide des biens volés ou autres biens personnels quand ils ne servent plus d'éléments de preuve;

le droit à un traitement rapide du cas et à être périodiquement informé de l'évolution des procédures judiciaires jusqu'à la décision finale; et quand les ressources et le personnel le permettent, le droit à être informé de la libération du contrevenant dans les cas d'infractions majeures; le droit pour les victimes de viol ou d'agression sexuelle d'être interrogées par une femme quand les ressources et le personnel le permettent» (IACP, 1983). 
Même si ces droits inaliénables ont été sanctionnés par les Nations Unies et inscrits dans la plupart des législatures américaines, les services de police du Québec n'ont pas encore intégré dans leurs lois, règlements et directives, une reconnaissance explicite des droits des victimes.

Il est temps que les responsables des services de police québécois «instaurent des procédures et forment le personnel de façon à reconnaître et à appliquer les «droits inaliénables de toutes les victimes d'actes criminels» (Waller, 1990). Il est dans l'intérêt des services de police de prendre des mesures et de trouver le personnel et les ressources nécessaires afin de fournir de l'aide et d'assurer la justice - y compris pour les victimes - dans les lieux publics et les foyers québécois».

Toutefois, il faut bien voir que les politiques recommandées par l'IACP sont parfois ambiguës ou restrictives («quand les ressources le permettent») et qu'elles ne tiennent pas compte des compétences et juridictions particulières aux pays et aux provinces.

\section{PROTÉGER LES INTÉRÊTS DES VICTIMES LORS DU PROCESSUS JUDICIAIRE}

Les victimes d'actes criminels subissent des traumatismes et des préjudices additionnels lorsqu'elles collaborent avec le système de justice pénale (Shapland, 1985; CCDS, 1985; Waller, 1988). En outre, les pratiques judiciaires négligent généralement leurs besoins en matière de réparation, de respect de leur vie privée, de sécurité personnelle, sans oublier les répercussions que le crime peut avoir dans leur vie (Waller, 1988).

La France (1986) et les pays qui se sont inspirés du modèle français ont adopté des mesures pour protéger à long terme les droits des victimes durant les procédures pénales. La France l'a fait en partie grâce à la loi qui permet aux victimes de se porter "partie civile» lors des procédures pénales, qui favorise le recours au dédommagement et informe indirectement les victimes sur l'enquête et les poursuites, en leur donnant l'occasion d'exprimer leur point de vue sur les décisions prises par l'État à l'égard du contrevenant. Mais ce qui distingue surtout la France des autres régimes de droit civil, c'est l'octroi d'une aide financière aux victimes incapables de se payer les services d'un avocat. Cette mesure fait de la partie civile une réalité concrète, car dans au moins la moitié des causes donnant lieu à procès, les victimes sont représentées par un procureur.

Une analyse de l'expérience vécue par les victimes en Grande-Bretagne (Shapland, 1985) a démontré la nécessité pour le système de justice pénale 
d'accorder plus d'importance aux intérêts des victimes. Les juges britanniques, plus particulièrement les magistrats à temps partiel, se sont mis à ordonner le dédommagement des victimes par les infracteurs à la suite des modifications dans la loi sur la justice pénale de 1973 qui ont été renforcées en 1982. Dans les tribunaux inférieurs, une condamnation pour dommages criminels entraîne une ordonnance de dédommagement dans à peu près les deux tiers des cas. Même dans les cas de crimes contre la personne, près d'un cinquième des jugements s'accompagnent d'une telle ordonnance. Curieusement, les Britanniques ont adopté ces ordonnances de dédommagement sans avoir pour autant prévu par des dispositions législatives des mécanismes permettant aux victimes d'informer directement la cour de leur besoin de dédommagement.

Aux États-Unis, les lois accordent de plus en plus aux victimes le droit d'être présentes et entendues lors des enquêtes sur cautionnement, au moment de la détermination de la peine et lors des audiences sur les libérations conditionnelles (NOVA, 1989). Les juges ont eux-mêmes élaboré des directives pour faciliter l'accès au système judiciaire, favoriser le dédommagement et permettre aux victimes de recevoir de l'information sur les programmes d'indemnisation et sur les services (Judicial Institute, 1984). Il existe un courant de plus en plus fort en faveur d un amendement constitutionnel qui garantirait la protection des intérêts des victimes dans les procédures pénales. En 1986, on formulait généralement cet amendement dans les termes suivants: «Les victimes d'actes criminels doivent bénéficier de certains droits élémentaires, y compris le droit à l'information, le droit d'être présentes et d'être entendues à chacune des étapes décisives de la procédure pénale fédérale ou d'État dans la mesure où ces droits ne contreviennent pas aux droits actuellement garantis par la constitution». (NOVA, 1988, 7).

Au Canada, les principes adoptés par le fédéral et les provinces établissent que lorsqu'il y a atteinte aux intérêts de la victime, il faut que l'opinion et les inquiétudes de celle-ci puissent être portées à l'attention du tribunal, dans la mesure où c'est approprié et conforme au droit criminel et à la procédure pénale (Canada, 1988). En outre, dans un rapport récent, un comité parlementaire sur la détermination de la peine (Daubney, 1988, 54-55) a établi que la sentence avait pour principal objectif de favoriser la préservation d'une société sûre en obligeant les délinquants - ou en les incitant à le faire «à reconnaître le tort qu'ils ont fait à la victime et à la société, et à assumer la responsabilité des conséquences de leur comportement».

Il reste encore beaucoup à faire pour sensibiliser les juges et les avocats aux besoins des victimes. On pourrait tenter l'expérience de tribunaux «unifiés» qui traiteraient en même temps de nombreuses causes civiles et criminelles comme en France. On pourrait ainsi réduire les coûts de l'appareil judi- 
ciaire et l'accumulation des dossiers en attente. Il faudrait étudier l'éventualité d'un amendement constitutionnel visant à protéger les intérêts des victimes (NOVA, 1989). Ce constat ne s'applique pas seulement au Québec. C'est la loi fédérale qu'il faut continuer à améliorer de concert avec les autres provinces.

\section{LES VICTIMES RÉCLAMENT DES PROGRAMMES DE PRÉVENTION}

Sans aucun doute, le moyen le plus efficace pour réduire les traumatismes psychologiques consécutifs à des actes criminels consiste à prévenir la criminalité. Les projets qui ont réussi à réduire certains types d'actes criminels se sont multipliés et on connaît de mieux en mieux les moyens de réduire les autres formes de crime (CCDS, 1984), bien qu'il s'agisse rarement de programmes portant sur un nombre significatif de collectivités. Les efforts enregistrés en Amérique du Nord pour développer des approches globales de lutte contre la consommation de drogue et les récents progrès réalisés en Europe sur la prévention du crime dans les municipalités semblent cependant prometteurs (Waller, 1989a; Waller, 1989b).

Les victimes d'actes criminels veulent être protégées du suspect ou du contrevenant et elles désirent vivre dans une société plus sûre et plus paisible. Pour cela, il faut accorder plus de place aux intérêts des victimes en adoptant des mesures efficaces de prévention (CCDS, 1984; Waller, 1989a).

Les victimes ont souvent besoin de savoir quelles mesures préventives elles peuvent prendre, par exemple pour assurer la sécurité de leur habitation. Il faudrait donc instaurer un service d'information fiable sur les mesures de protection à prendre. Même dans le cas où les services policiers se sont dotés d'une unité spéciale d'intervention auprès des victimes, ces dernières ne bénéficient pas nécessairement de services de qualité sur ce plan.

Les victimes de crimes contre la personne, en particulier celles qui connaissent le suspect, veulent savoir comment éviter d'éventuelles représailles de la part de ce dernier. Elles peuvent aussi faire l'objet de menaces et d'intimidation visant à les dissuader de témoigner. Dans des cas exceptionnels, la police peut leur offrir une nouvelle identité et une nouvelle adresse; mais en général, on ne fait pas grand-chose pour assurer leur protection.

Les victimes bénéficieraient d'une meilleure protection si on utilisait l'information recueillie par la police pour s'attaquer aux facteurs qui alimentent la criminalité. Si la police utilisait ces données pour encourager les services de logement, les écoles, les services d'urbanisme et les services sociaux à 
améliorer leurs programmes, il y aurait moins de crimes (Waller, 1989a, 1989b).

Il est grand temps de s'occuper de prévention. Les maires, les décideurs et administrateurs municipaux ainsi que les urbanistes doivent prendre des mesures pour modifier leurs programmes d'habitation et de services sociaux, leurs programmes récréatifs, leurs politiques d'emploi, les services de police et les programmes de protection de l'enfance afin d'améliorer la sécurité des villes et des collectivités dans les dix prochaines années. La Conférence européenne et nord-américaine sur la sécurité et la prévention de la criminalité en milieu urbain, qui s'est tenue à Montréal du 10 au 13 octobre 1989, a indiqué des pistes concrètes en ce sens (FCM, 1990). La décision de la Fédération des municipalités canadiennes de travailler en collaboration avec la conférence des maires des États-Unis et le Forum européen sur la sécurité urbaine pour amorcer une coopération entre les maires d'Europe de l'Ouest et d'Amérique du Nord afin d'améliorer la sécurité des villes constitue un important point de départ.

\section{CONCLUSION}

Le Québec a déjà posé des gestes très importants pour faire de la Charte des Nations Unies pour la défense des droits des victimes d'actes criminels une réalité dans la province.

Les programmes d'aide et d'indemnisation des victimes mis sur pied jusqu'ici ont été amorcés de façon prudente et expérimentale. Le recours à la plupart de ces programmes demeure facultatif; ce qui explique qu'on n'en ait pas fait jusqu'à maintenant un usage systématique. Il faut maintenant profiter de l'expérience acquise pour élargir et consolider ces programmes.

Si on demandait aux policiers et au ministère public d'informer les victimes sur les programmes existants, l'État pourrait indemniser toutes les personnes admissibles. Si on renforçait le programme INFOVAC, les victimes pourraient se familiariser avec le processus pénal. Et les victimes bénéficieraient d'une aide plus complète si le Québec professionnalisait davantage ses programmes d'aide et exploitait son avance en matière de services en santé mentale pour victimes d'actes criminels afin d'élaborer des lignes directrices à l'intention des policiers, du personnel des services sociaux et des professionnels de la santé.

Mais ces progrès relèvent en grande part de l'initiative des services de police: ce sont eux qui peuvent, après le crime, informer les victimes des services disponibles et les diriger vers les organismes d'aide. Sur le plan 
budgétaire, il ne s'agit pas nécessairement de projets coûteux. Si les dirigeants des services de police décidaient de prioriser, parmi les besoins de la collectivité, l'aide aux victimes d'actes criminels, ils favoriseraient les changements de mentalité nécessaires chez les agents de police.

Les victimes, lorsque leurs intérêts sont en jeu, pourraient être entendues et respectées dans les cours criminelles si le Canada et le Québec adoptajent des lois aussi avancées que celles qui se répandent actuellement aux États-Unis ou qui sont déjà en vigueur en France. Si on suivait l'exemple britannique ainsi que les principes de Daubney (1988) et de la loi C-89 (1989), les ordonnances de dédommagement des victimes par les contrevenants deviendraient plus répandues en matière de sentences.

Mais il est aussi important pour les personnes qui ont déjà été victimes d'actes criminels que le gouvernement fédéral, le gouvernement provincial et les administrations municipales s'engagent à mettre en ouvre le programme «Pour des villes plus sûres», ce qui diminuerait le taux de victimisation d'ici l'an 2000 (FCM, 1990).

Bref, si l'on veut répondre de mieux en mieux aux besoins des victimes, les prochaines étapes pour le Québec au cours des années 90 devront impliquer une plus grande mobilité des services de police, une amélioration du processus judiciaire et un accent sur la prévention du crime, particulièrement au niveau des municipalités.

\section{RÉFÉRENCES}

AMERNIC, Jerry (1984). Victims: the Orphans of Justice. Toronto, McClelland \& Stewart.

BARIL, Micheline et S. CUSSON (1989). «La déclaration de la victime». Montréal, allocution de Plaidoyer-Victimes.

BASSIOUNI, M. CHERIF (1988), «International Protection of Victims». Nouvelles Études Pénales VII, numéro spécial.

C-89 (1989). Loi modifiant le Code criminel (victimes d'actes criminels).

CANADA (1988). «Principes fondamentaux de justice pour les victimes d'actes criminels», Entente fédérale-provinciale-territoriale. Saskatoon, mars 1988.

CANADA (1986). Indemnisation des victimes d'actes criminels 1986 , Justice. Ottawa, Statistique Canada.

CANADA (1983a). Groupe d'étude fédéral-provincial canadien sur la justice pour les vic. times d'actes criminels, Rapport, Ottawa, Approvisionnements et Services.

CANADA (1983b). Les victimes d'actes criminets. Bulletin n 1 , Ottawa, Solliciteur général du Canada. 
CCDS (1985). L'Hygiène mentale des victimes de crime et leurs familles, Ottawa, Conseil canadien de développement social.

CCDS (1984). La prévention du crime par le développement social, Ottawa, Conseil canadien de développement social.

CCDS (1981). Les victimes $d$ ' actes criminels : droits et services, Ottawa, Conseil canadien de développement social.

DAUBNEY, David (1988). Des responsabilités à assumer, Ottawa, Chambre des communes.

EDMONTON POLICE DEPARTMENT (1989). «Victim Services Unit: a Summary».

FCM (1990). Pour des villes plus sâres, Ottawa, Fédération canadienne des muricipalités.

FRANCE (1986). Guide des droits des victimes, ministère de la Justice, Paris, Gallimard.

INAVEM (1986-). Bulletin de nouvelles, Paris, Institut national d'ajde aux victimes et médiation.

IACP (1983). «Crime Victims Bill of Rights», Board of Officers, Arlington, International Association of Chiefs of Police, Policy Center.

JAFFE, Peter et al. (1984). «Evaluating the Impact of a Specialized Civilian Family Crisis Unit Within a Police Force on the Resolution of Family Conflicts", Journal of Preventive Psychiatry, 2:1:63-73.

JUDICIAL INSTITUTE (1984). Guidelines for the Judiciary on the Treatment of Victims.

Liaison (1982 8:4. p. 26).

MacLEOD, Linda (1989), «Espoirs et déceptions dans le domaine des femmes battues: Progrès, dilemmes et perspectives de prévention», document de travail, Ottawa, Santé nationale et bien-être social.

MAGUIRE, Mike et Claire CORBETT (1987). The Effects of Crime and the Work of Victims Support Schemes, Brookfield, Gower

MANITOBA (1986). Loi 30 - Les droits des victimes d'actes criminels.

NOVA (1989). Victim Rights and Services: a Legistative Directory, Washington, D.C., National Organization for Victim Assistance.

ONTARIO (1989). Loi sur les services de police.

ONU (1985). Déclaration des principes fondamentaux de justice relatifs aux victimes de la criminalité et aux victimes d'abus de pouvoir, Organisation des Nations Unies, Assemblée générale A/RES/40/34.

PLAIDOYER-VICTIMES INC. (1988). Guide d'intervention auprès des victimes d'actes criminels.

PULLYBLANK, John (1986). The Victim Services Unit of the Edmonton Police Department : an Evaluation, Ottawa, Solliciteur général.

QUÉBEC (1989). «L'aide aux victimes d'actes criminels: fiction ou réalité». Allocution du sous-ministre de la Justice, Montréal, 25 octobre.

QUÉBEC (1988). Loi 8 sur l'aide aux victimes d'actes criminels.

RIZKALLA, Samir (éd.) (1983). Crime, victime et communauté, Montréal, Société de criminologie du Québec.

SHAPLAND, Joanna et J. W. P. DUFF (1985). Victims in the Criminal Justice System, London, Gower, p. 175-194.

USA (1988). Report to the Nation on Crime and Justice, Washington, DC, Department of Justice, U.S. Government Printing Office. 
WALLER, Irvin (1990). «The Police: First in Aid", in Lurigio, Arthur, W. G. Skogan \& R. C. Davies (ed), Victim of Crime: Problems, Policies, Programs. Newbury Park, Sage.

WALLER, Irvin (1989a). Tendances actuelles de la prévention du crime en Europe: Répercussions au Canada, Ottawa, Justice Canada.

WALLER, Irvin (1989b). «Crime Prevention in a Community Policing Context», Ottawa, Rapport au Solliciteur Général du Canada.

WALLER, Irvin (1988). Le rôle de la victime dans la détermination de la peine et les processus connexes, Ottawa, Justice Canada.

WALLER. Irvin (1985). «Le stress après le crime: sa nature et les besoins qu'il exige», in Documents de travail, Conférence sur les traumatismes propres aux victimes de crime, Ottawa, Conseil canadien de développement social.

WALLER, Irvin (1983). «Les victimes d'actes criminels: besoins, services et réformes Les oubliées de la politique sociale», in Rizkalla (19830. p. 182-240.

Pour plus de renseignements:

* Le Centre national de documentation sur les victimes - Justice Canada, K IA 0H8 1-800-267-0454.

* Le Centre national d'information sur la violence dans la famille - 1-800-267-1291. 